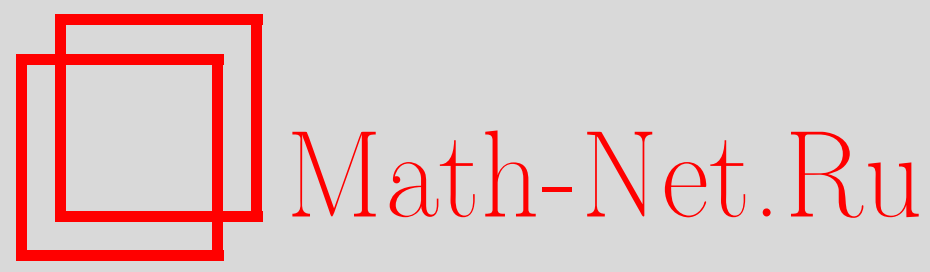

Д. В. Горбачев, В. И. Иванов, Одна экстремальная задача для многочленов, связанная с кодами и дизайнами, Матем. заметки, 2000, том 67, выпуск 4, 508-513

DOI: https://doi.org/10.4213/mzm865

Использование Общероссийского математического портала Math-Net.Ru подразумевает, что вы прочитали и согласны с пользовательским соглашением http://www.mathnet.ru/rus/agreement

Параметры загрузки:

IP : 54.157 .27 .8

26 апреля 2023 г., $13: 22: 26$

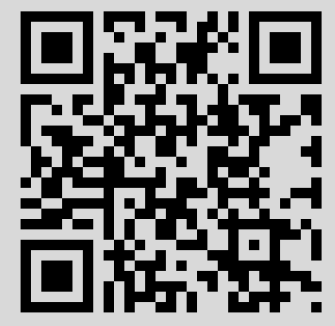




\title{
ОДНА ЭКСТРЕМАЛЬНАЯ ЗАДАЧА ДЛЯ МНОГОЧЛЕНОВ, СВЯЗАННАЯ С КОДАМИ И ДИЗАЙНАМИ
}

\author{
Д. В. Горбачев, В. И. Иванов
}

Решается одна экстремальная задача В. А. Юдина для алгебраических многочленов, связанная с кодами и дизайнами.

Библиография: 6 названий.

В связи с экстремальной задачей нахождения среди кодов дизайна наибольшего порядка В. А. Юдин [1] рассмотрел следующую экстремальную задачу для многочленов. Найти величину

$$
B_{s}(\sigma)=\sup _{h \in H_{s}(\sigma)}\{\lambda \in[-1,1]: h(t) \geqslant 0,-1 \leqslant t \leqslant \lambda\}
$$

где $s \in \mathbb{N}, H_{s}(\sigma)=\left\{h(t)=\sum_{\nu=1}^{s} \widehat{h}_{\nu} P_{\nu}(t): \widehat{h}_{\nu} \geqslant 0\right\},\left\{P_{\nu}\right\}$ - система многочленов, ортогональная на $[-1,1]$ относительно некоторой меры $d \sigma, P_{\nu}(1)=1$. Им была получена оценка сверху для четного $s=2 q$ :

$$
B_{2 q}(\sigma) \leqslant t_{2}\left(P_{q+1}\right)
$$

где $1>t_{1}\left(P_{q+1}\right)>t_{2}\left(P_{q+1}\right)>\cdots>t_{q+1}\left(P_{q+1}\right)>-1-$ нули $P_{q+1}$, и в качестве экстремального многочлена был предложен многочлен

$$
h_{2 q}(t)=\frac{P_{q+1}^{2}(t)}{\left(t-t_{1}\left(P_{q+1}\right)\right)\left(t-t_{2}\left(P_{q+1}\right)\right)}
$$

для которого не удалось доказать положительность коэффициентов Фурье.

Теорема 1. Пусть $\left\{Q_{\nu}\right\}$ - система многочленов, ортогональная на $[-1,1]$ относительно меры $(1+t) d \sigma, Q_{\nu}(1)=1$. Тогда для нечетного $s=2 q+1$ справедлива оченка сверху

$$
B_{2 q+1}(\sigma) \leqslant t_{2}\left(Q_{q+1}\right)
$$

Работа выполнена при финансовой поддержке Российского фонда фундаментальных исследований (проект № 97-01-00318) и Минобразования (проект № 97-0-1.4-78) 
ДоказАтеЛьство. Аналогично доказательству теоремы 3 из [1]. Пусть $t_{k}=$ $t_{k}\left(Q_{q+1}\right)$. Предположим обратное: существует многочлен $h \in H_{2 q+1}(\sigma)$, неотрищательный на $[-1, \lambda]$, где $\lambda \in\left(t_{2}, 1\right]$. Тогда возможны 3 случая:

1) $\lambda \in\left[t_{1}, 1\right]$;

2) $\lambda \in\left(t_{2}, t_{1}\right)$ и $h\left(t_{1}\right) \geqslant 0$;

3) $\lambda \in\left(t_{2}, t_{1}\right)$ и $h\left(t_{1}\right)<0$.

Из $\widehat{h}_{0}=0, \widehat{h}_{1} \geqslant 0, P_{0}(t)=1$ и $P_{1}(t)=\alpha t+1-\alpha$, где $\alpha>1 / 2\left(\right.$ так как $t_{1}\left(P_{1}\right)=(\alpha-1) / \alpha$ $\in(-1,1))$, следует, что $\int_{-1}^{1} h d \sigma=0$ и $\int_{-1}^{1}(\lambda-t) h d \sigma \leqslant 0$.

Воспользуемся квадратурной формулой Гаусса с фиксированным узлом [2]

$$
\int_{-1}^{1} h(t) d \sigma=A_{0} h(-1)+\sum_{k=1}^{q+1} A_{k} h\left(t_{k}\right), \quad A_{k}>0,
$$

справедливой для многочленов $h$ степени $2 q+2$. Тогда в случаях 1) и 2$)$ имеем

$$
0=A_{0} h(-1)+\sum_{k=1}^{q+1} A_{k} h\left(t_{k}\right)
$$

где все слагаемые неотрицательны. Следовательно $h(-1)=0, h\left(t_{k}\right)=0(k=\overline{1, q+1})$, при этом в точках $t_{k}(k=\overline{2, q+1})$ многочлен $h$ в силу неотрицательности имеет по крайней мере двойные нули. Получили, что многочлен $h$ степени $2 q+1$ имеет по крайней мере $1+2 q+1=2 q+2$ нулей, что невозможно.

В случае 3$)$ напишем (3) для многочлена $(\lambda-t) h(t)$ степени $2 q+2$

$$
0 \geqslant \int_{-1}^{1}(\lambda-t) h(t) d \sigma=A_{0}(\lambda+1) h(-1)+\sum_{k=1}^{q+1} A_{k}\left(\lambda-t_{k}\right) h\left(t_{k}\right),
$$

где $(\lambda+1) h(-1) \geqslant 0,\left(\lambda-t_{k}\right) h\left(t_{k}\right) \geqslant 0(k=\overline{2, q+1})$ и $\left(\lambda-t_{1}\right) h\left(t_{1}\right)>0$, что невозможно. Теорема доказана.

Отметим, что при доказательстве оценки (2) используется только равенство нулю нулевого и неотрицательность первого коэффициента Фурье. В качестве экстремального многочлена в оценке (2) может быть предложен многочлен

$$
h_{2 q+1}(t)=\frac{(1+t) Q_{q+1}^{2}(t)}{\left(t-t_{1}\left(Q_{q+1}\right)\right)\left(t-t_{2}\left(Q_{q+1}\right)\right)},
$$

у которого нулевой и первый коэффициенты Фурье равны нулю (в силу ортогональности $Q_{q+1}$ любому многочлену степени $q$ ).

Во многих случалх удается доказать принадлежность многочленов $h_{s}$ к $H_{s}(\sigma)$ и тем самым установить точность приведенных оценок $B_{s}(\sigma)$. Основополагающей является следуюшая лемма, имеющая и самостоятельное значение. 
ЛЕмма. Пусть $d_{\nu}=\left(\int_{-1}^{1} P_{\nu}^{2} d \sigma\right)^{-1}, t_{k}=t_{k}\left(P_{q+1}\right)$,

$$
\frac{P_{q+1}(t)}{\left(t-t_{1}\right)\left(t-t_{2}\right)}=\sum_{\nu=0}^{q-1} d_{\nu} a_{\nu} P_{\nu}(t)
$$

Toгдa

$$
a_{0} \geqslant a_{1} \geqslant \cdots \geqslant a_{q-1} \geqslant 0 .
$$

ДоКАЗАТЕЛЬСТВО. Из тождества

$$
\frac{1}{\left(t-t_{1}\right)\left(t-t_{2}\right)}=\frac{1}{t_{1}-t_{2}}\left(\frac{1}{t-t_{1}}-\frac{1}{t-t_{2}}\right)
$$

и формулы Кристоффеля-Дарбу [3]

$$
\sum_{\nu=0}^{n} d_{\nu} P_{\nu}(x) P_{\nu}(y)=C_{n} \frac{P_{n+1}(x) P_{n}(y)-P_{n}(x) P_{n+1}(y)}{x-y}, \quad C_{n}>0
$$

следует, что

$$
a_{\nu}=B_{q}^{-1}\left(P_{q}\left(t_{1}\right) P_{\nu}\left(t_{2}\right)-P_{\nu}\left(t_{1}\right) P_{q}\left(t_{2}\right)\right), \quad \nu=\overline{0, q}, \quad a_{q}=0
$$

где $B_{q}=-\left(t_{1}-t_{2}\right) C_{q} P_{q}\left(t_{1}\right) P_{q}\left(t_{2}\right)$. Известно [3], что

$$
\begin{gathered}
P_{i}\left(t_{1}\right)>0, \quad i=\overline{0, q}, \quad P_{i}\left(t_{2}\right) \geqslant 0, \\
i=\overline{0, i_{1}-1}, \quad P_{i}\left(t_{2}\right)<0, \quad i=\overline{i_{1}, q},
\end{gathered}
$$

для некоторого $0<i_{1} \leqslant q$. Отсюда $B_{q}>0$.

Покажем, что для $0 \leqslant i \leqslant j \leqslant q$ выполнено неравенство

$$
P_{j}\left(t_{1}\right) P_{i}\left(t_{2}\right) \geqslant P_{i}\left(t_{1}\right) P_{j}\left(t_{2}\right) .
$$

Вначале установим неравенство

$$
P_{i}\left(t_{1}\right) P_{i-1}\left(t_{2}\right) \geqslant P_{i-1}\left(t_{1}\right) P_{i}\left(t_{2}\right), \quad 0<i \leqslant q .
$$

Из (4) следует, что

$$
P_{i}\left(t_{1}\right) P_{i-1}\left(t_{2}\right)-P_{i-1}\left(t_{1}\right) P_{i}\left(t_{2}\right)=\frac{t_{1}-t_{2}}{C_{i-1}} \sum_{k=0}^{i-1} d_{k} P_{k}\left(t_{1}\right) P_{k}\left(t_{2}\right) .
$$

Отсюда и из (6) вытекает оценка

$$
P_{i}\left(t_{1}\right) P_{i-1}\left(t_{2}\right)-P_{i-1}\left(t_{1}\right) P_{i}\left(t_{2}\right) \geqslant 0, \quad 0<i \leqslant i_{1}
$$




$$
\begin{gathered}
P_{i}\left(t_{1}\right) P_{i-1}\left(t_{2}\right)-P_{i-1}\left(t_{1}\right) P_{i}\left(t_{2}\right) \geqslant \frac{t_{1}-t_{2}}{C_{i-1}} \sum_{k=0}^{q} d_{k} P_{k}\left(t_{1}\right) P_{k}\left(t_{2}\right) \\
=\frac{C_{q}}{C_{i-1}}\left(P_{q+1}\left(t_{1}\right) P_{q}\left(t_{2}\right)-P_{q}\left(t_{1}\right) P_{q+1}\left(t_{2}\right)\right)=0, \quad i_{1}<i \leqslant q .
\end{gathered}
$$

Докажем (7) индукцией по $i=j, j-1, \ldots, 0$. При $i=j$ неравенство (7) верно. Пусть оно верно при $0<i \leqslant j$. Тогда, учитывая $(6),(8)$, получим

$$
\begin{aligned}
P_{j}\left(t_{1}\right) P_{i-1}\left(t_{2}\right) & =\frac{P_{j}\left(t_{1}\right)}{P_{i}\left(t_{1}\right)} P_{i}\left(t_{1}\right) P_{i-1}\left(t_{2}\right) \geqslant \frac{P_{j}\left(t_{1}\right)}{P_{i}\left(t_{1}\right)} P_{i-1}\left(t_{1}\right) P_{i}\left(t_{2}\right) \\
& =\frac{P_{i-1}\left(t_{1}\right)}{P_{i}\left(t_{1}\right)} P_{j}\left(t_{1}\right) P_{i}\left(t_{2}\right) \geqslant \frac{P_{i-1}\left(t_{1}\right)}{P_{i}\left(t_{1}\right)} P_{i}\left(t_{1}\right) P_{j}\left(t_{2}\right)=P_{i-1}\left(t_{1}\right) P_{j}\left(t_{2}\right)
\end{aligned}
$$

и неравенство (7) верно с $i-1$ вместо $i$.

Из $(4),(5)$, учитьвая $P_{\nu}(1)=1, P_{q+1}\left(t_{k}\right)=0$ и $(7)$, получим для $0 \leqslant \nu<q$

$$
\begin{aligned}
\frac{B_{q} C_{\nu} C_{q}\left(a_{\nu}-a_{\nu+1}\right)}{\left(1-t_{1}\right)\left(1-t_{2}\right)}= & C_{q} \frac{P_{q}\left(t_{1}\right)-P_{q+1}\left(t_{1}\right)}{1-t_{1}} C_{\nu} \frac{P_{\nu}\left(t_{2}\right)-P_{\nu+1}\left(t_{2}\right)}{1-t_{2}} \\
& -C_{\nu} \frac{P_{\nu}\left(t_{1}\right)-P_{\nu+1}\left(t_{1}\right)}{1-t_{1}} C_{q} \frac{P_{q}\left(t_{2}\right)-P_{q+1}\left(t_{2}\right)}{1-t_{2}} \\
= & \sum_{j=0}^{q} d_{j} P_{j}\left(t_{1}\right) \sum_{i=0}^{\nu} d_{i} P_{i}\left(t_{2}\right)-\sum_{i=0}^{\nu} d_{i} P_{i}\left(t_{1}\right) \sum_{j=0}^{q} d_{j} P_{j}\left(t_{2}\right) \\
= & \sum_{i=0}^{\nu} \sum_{j=\nu+1}^{q} d_{i} d_{j}\left(P_{j}\left(t_{1}\right) P_{i}\left(t_{2}\right)-P_{i}\left(t_{1}\right) P_{j}\left(t_{2}\right)\right) \geqslant 0 .
\end{aligned}
$$

Таким образом, $a_{0} \geqslant a_{1} \geqslant \cdots \geqslant a_{q-1} \geqslant a_{q}=0$. Лемма доказана.

Теорема 2. Eсли $P_{i}(t) P_{j}(t)=\sum_{k=|i-j|}^{i+j} p_{i j k} P_{k}(t)$, zдe $p_{i j k} \geqslant 0$, mo $h_{2 q} \in H_{2 q}(\sigma)$ и, следовательно,

$$
B_{2 q}(\sigma)=t_{2}\left(P_{q+1}\right) \text {. }
$$

ДокАЗАТЕЛЬСТво очевидно.

TEOPEMA 3. Ecлu

a) $Q_{i}(t) Q_{j}(t)=\sum_{k=|i-j|}^{i+j} q_{i j k} Q_{k}(t)$, где $q_{i j k} \geqslant 0$, илu

б) $P_{i}(t) P_{j}(t)=\sum_{k=|i-j|}^{i+j} p_{i j k} P_{k}(t)$, где $p_{i j k} \geqslant 0 u P_{\nu}(-t)=(-1)^{\nu} P_{\nu}(t)$, то $h_{2 q+1} \in H_{2 q+1}(\sigma)$ и, следовательно,

$$
B_{2 q+1}(\sigma)=t_{2}\left(Q_{q+1}\right)
$$


ДОКАЗАТЕЛЬСТво. Пусть $P_{\nu}(-1)=\varepsilon_{\nu}$. Известно [4], что $\operatorname{sgn} \varepsilon_{\nu}=(-1)^{\nu}$ и

$$
Q_{\nu}(t)=\frac{2}{C_{\nu}\left(\varepsilon_{\nu}-\varepsilon_{\nu+1}\right)} \sum_{i=0}^{\nu} d_{i} \varepsilon_{i} P_{i}(t)=\frac{2\left(P_{\nu+1}(t) \varepsilon_{\nu}-P_{\nu}(t) \varepsilon_{\nu+1}\right)}{\left(\varepsilon_{\nu}-\varepsilon_{\nu+1}\right)(1+t)} .
$$

Отсюда

$$
(1+t) Q_{\nu}(t)=\alpha_{\nu} P_{\nu}(t)+\beta_{\nu} P_{\nu+1}(t), \quad \alpha_{\nu}, \beta_{\nu} \geqslant 0 .
$$

a) Пусть $Q_{i}(t) Q_{j}(t)=\sum_{k=|i-j|}^{i+j} q_{i j k} Q_{k}(t), q_{i j k} \geqslant 0$. По теореме 2

$$
\frac{Q_{q+1}^{2}(t)}{\left(t-t_{1}\left(Q_{q+1}\right)\right)\left(t-t_{2}\left(Q_{q+1}\right)\right)}=\sum_{\nu=1}^{2 q} \gamma_{\nu} Q_{\nu}(t), \quad \gamma_{\nu} \geqslant 0
$$

Отсюда и из (9) следует, что

$$
h_{2 q+1}(t)=\sum_{\nu=1}^{2 q} \gamma_{\nu}\left(\alpha_{\nu} P_{\nu}(t)+\beta_{\nu} P_{\nu+1}(t)\right)=\sum_{\nu=1}^{2 q+1}\left(\widehat{h}_{2 q+1}\right)_{\nu} P_{\nu}(t), \quad\left(\widehat{h}_{2 q+1}\right)_{\nu} \geqslant 0 .
$$

б) Пусть $P_{i}(t) P_{j}(t)=\sum_{k=|i-j|}^{i+j} p_{i j k} P_{k}(t), p_{i j k} \geqslant 0$ и $P_{\nu}(-t)=(-1)^{\nu} P_{\nu}(t) ; e_{\nu}=$ $\left(\int_{-1}^{1} Q_{\nu}^{2}(t)(1+t) d \sigma\right)^{-1}$. Тогда $\varepsilon_{\nu}=(-1)^{\nu}$,

$$
\begin{aligned}
Q_{\nu}(t) & =C_{\nu}^{-1} \sum_{i=0}^{\nu} d_{i}(-1)^{i+\nu} P_{i}(t)=\frac{P_{\nu}(t)+P_{\nu+1}(t)}{1+t} \\
e_{\nu} & =\left(\int_{-1}^{1} C_{\nu}^{-1} \sum_{i=0}^{\nu} d_{i}(-1)^{i+\nu} P_{i}(t)\left(P_{\nu}(t)+P_{\nu+1}(t)\right) d \sigma(t)\right)^{-1}=C_{\nu} .
\end{aligned}
$$

Пусть

$$
\frac{Q_{q+1}(t)}{\left(t-t_{1}\left(Q_{q+1}\right)\right)\left(t-t_{2}\left(Q_{q+1}\right)\right)}=\sum_{k=0}^{q-1} e_{k} b_{k} Q_{k}(t)=\sum_{i=0}^{q-1} \delta_{i} P_{i}(t) .
$$

По лемме $b_{0} \geqslant b_{1} \geqslant \cdots \geqslant b_{q-1} \geqslant 0$. Отсюда и из (10) вытекает, что

$$
\sum_{k=0}^{q-1} e_{k} b_{k} Q_{k}(t)=\sum_{k=0}^{q-1} b_{k} \sum_{i=0}^{k} d_{i}(-1)^{i+k} P_{i}(t)=\sum_{i=0}^{q-1} d_{i} P_{i}(t) \sum_{k=i}^{q-1}(-1)^{i+k} b_{k}
$$

и $\delta_{i}=d_{i} \sum_{k=i}^{q-1}(-1)^{i+k} b_{k}=d_{i}\left(b_{i}-b_{i+1}+\cdots\right) \geqslant 0$. Таким образом,

$$
\begin{aligned}
\frac{(1+t) Q_{q+1}^{2}(t)}{(t}- & \left.t_{1}\left(Q_{q+1}\right)\right)\left(t-t_{2}\left(Q_{q+1}\right)\right) \\
= & \sum_{i=0}^{q-1} \delta_{i} P_{i}(t)\left(P_{q+1}(t)+P_{q+2}(t)\right) \\
= & \sum_{i=0}^{q-1} \delta_{i} \sum_{k=|q+1-i|}^{q+1+i} p_{i, q+1, k} P_{k}(t)+\sum_{i=0}^{q-1} \delta_{i} \sum_{k=|q+2-i|}^{q+2+i} p_{i, q+2, k} P_{k}(t) \\
= & \sum_{k=2}^{2 q} P_{k}(t) \sum_{i=|q+1-k|}^{q-1} \delta_{i} p_{i, q+1, k}+\sum_{k=3}^{2 q+1} P_{k}(t) \sum_{i=|q+2-k|}^{q-1} \delta_{i} p_{i, q+2, k} \\
= & \sum_{\nu=2}^{2 q+1}\left(\widehat{h}_{2 q+1}\right)_{\nu} P_{\nu}(t),
\end{aligned}
$$


где $\left(\widehat{h}_{2 q+1}\right)_{\nu} \geqslant 0$. Теорема доказана.

Применим полученные результаты к некоторым семействам ортогональных многочленов. Рассмотрим многочлены Якоби [3] $\left\{P_{\nu}^{\alpha, \beta}\right\}\left(d \sigma=(1-t)^{\alpha}(1+t)^{\beta} d t(\alpha, \beta>-1)\right.$, $\left.Q_{\nu}=P_{\nu}^{\alpha, \beta+1}\right)$. Известно [5], что $P_{i}^{\alpha, \beta}(t) P_{j}^{\alpha, \beta}(t)=\sum_{k=|i-j|}^{i+j} p_{i j k}^{\alpha, \beta} P_{k}(t)$, где $p_{i j k}^{\alpha, \beta} \geqslant 0$ при $\alpha \geqslant \beta \geqslant-1 / 2 ; P_{\nu}^{\alpha, \alpha}(-t)=(-1)^{\nu} P_{\nu}^{\alpha, \alpha}(t)$. Отсюда следует, что теорема 2 справедлива при $\alpha \geqslant \beta \geqslant-1 / 2$, теорема 3 - при $\alpha \geqslant \beta+1>0$ или $\alpha=\beta \geqslant-1 / 2$. (Интересно было бы доказать теорему 3 при $\beta<\alpha<\beta+1$ ). Таким образом, полное решение задачи (1) получено для многочленов Гегенбауэра $(\alpha=\beta \geqslant-1 / 2)$.

Теоремы 2 и 3 справедливы для некоторых семейств дискретных многочленов, например, многочленов Кравчука [4] $\left\{P_{\nu}(t)=K_{\nu}^{n, r}((n / 2)(1-t))\right\}(n, r \in \mathbb{N}, r \geqslant 2$, $\sum_{d=0}^{n}(r-1)^{d}\left(\begin{array}{l}n \\ d\end{array}\right) K_{i}^{n, r}(d) K_{j}^{n, r}(d)=0$ при $\left.i \neq j, Q_{\nu}(t)=K_{\nu}^{n-1, r}\left(\frac{n}{2}(1-t)\right)\right)$, поскольку известно [4], что $K_{i}^{n, r}(d) K_{j}^{n, r}(d)=\sum_{k=|i-j|}^{i+j} p_{i j k}^{n, r} K_{k}^{n, r}(d), p_{i j k}^{n, r} \geqslant 0$.

Пусть $n \in \mathbb{N}, n \geqslant 2, d \sigma=\left(1-t^{2}\right)^{(n-3) / 2} d t$. Юдин [1] показал, что любой $\cos B_{s}(\sigma)$-код $C$ на сфере $S^{n-1}$ (т.е. $\min _{x \neq y \in C} d(x, y)=\cos B_{s}(\sigma), d(x, y)=\arccos x y$ ) является дизайном порядка, не превосходящего $s-1$ (общее понятия кода и дизайна см. в [6]). Поскольку в этом случае мере $d \sigma$ соответствуют многочлены Гегенбауэра $P_{\nu}^{(n-3) / 2,(n-3) / 2}$, то окончательно получаем следуюший результат: любой $d_{s}\left(S^{n-1}\right)$-код $C \subset S^{n-1}$, где $d_{2 q}\left(S^{n-1}\right)=\cos t_{2}\left(P_{q+1}^{(n-3) / 2,(n-3) / 2}\right)$ и $d_{2 q+1}\left(S^{n-1}\right)=\cos t_{2}\left(P_{q+1}^{(n-3) / 2,(n-1) / 2}\right)$, является дизайном порядка, не превосходящего $s-1$.

Аналогичные результаты справедливы для многих FDNDF-полиномиальных по классификации Левенштейна [6] компактных метрических пространств. В частности, для пространства Хемминга $\mathbb{F}_{r}^{n}[6]$ имеет место следующее утверждение: любой $d_{s}\left(\mathbb{F}_{r}^{n}\right)$-код $C \subset \mathbb{F}_{r}^{n}$, где $d_{2 q}\left(\mathbb{F}_{r}^{n}\right)=\left\lceil t_{2}\left(K_{q+1}^{n, r}\right)\right\rceil$ и $d_{2 q+1}\left(\mathbb{F}_{r}^{n}\right)=\left\lceil t_{2}\left(K_{q+1}^{n-1, r}\right)\right\rceil(\lceil x\rceil$ - наименьшее целое число, превосходящее $x)$, является дизайном порядка, не превосходящего $s-1$. В некоторых случаях эта оценка достигается. Например, поскольку $t_{2}\left(K_{3}^{n, 2}\right)=n / 2$ и $d_{4}\left(\mathbb{F}_{2}^{n}\right)=\lceil n / 2\rceil$, то для кодов Адамара $[6]$ в $\mathbb{F}_{2}^{n}(n=4 l, l \in \mathbb{N})$, являющихся $n / 2$-кодами и дизайнами порядка 3 , имеем правильную оценку $3 \leqslant 3$.

\section{СПИСОК ЦИТИРОВАННОЙ ЛИТЕРАТУРЫ}

[1] Юдин В. А. Код и дизайн // Дискретная матем. 1997. Т. 9. №2. С. 3-11.

[2] Крылов В. И. Приближенное вычисление интегралов. М.: Физматгиз, 1959.

[3] Сегё Г. Ортогональные многочлены. М.: Физматгиз, 1962.

[4] Левенштейн В. И. Границы для упаковок метрических пространств и некоторые их приложения // Проблемы кибернетики. 1983. Т. 40. С. 43-110.

[5] Gasper G. Linearization of the product of Jacobi polynomials. I // Canad. J. Math. 1970. V. 2. №1. P. 171-175.

[6] Levenshtein V. I. On designs in compact metric spaces and a universal bound on their size // Discrete Math. 1998. V. 192. P. 251-271.

Тульский государственный университет

E-mail: dvg@mechmat.tsu.tula.ru, ivi@mechmat.tsu.tula.ru 